\title{
Comparative validity of microalbuminuria versus clinical mortality scores to predict pediatric intensive care unit outcomes
}

\author{
Shifa Nismath, $M D^{1}$, Suchetha S. Rao, MD¹, B.S. Baliga, MD¹, Vaman Kulkarni, MD², Gayatri M. Rao, $\mathrm{PhD}^{3}$ \\ ${ }^{1}$ Department of Paediatrics, Kasturba Medical College Mangalore, Manipal Academy of Higher Education, Manipal Karnataka, India; ${ }^{2}$ Community Medicine, Kasturba \\ Medical College Mangalore, Manipal Academy of Higher Education, Manipal Karnataka, India; ${ }^{3}$ Biochemistry, Kasturba Medical College Mangalore, Manipal Academy \\ of Higher Education, Manipal Karnataka, India
}

Background: Predicting the prognosis of patients admitted to the pediatric intensive care unit (PICU) is very important in determining further management and resource allocation. The prognostication of critically ill children can be challenging; hence, accurate methods for predicting outcomes are needed.

Purpose: To evaluate the role of microalbuminuria at admission as a prognostic marker in comparison to standard Pediatric Risk of Mortality (PRISM) and Pediatric Logistic Organ Dysfunction (PELOD) mortality scores in children admitted to the PICU.

Methods: This cross-sectional study was conducted from January 2015 to October 2016. Eighty-four patients aged 1 month to 18 years admitted to the PICU of teaching hospitals for more than 24 hours were enrolled by convenience sampling method. Microalbuminuria was estimated by spot urinary albumin-creatinine ratio. PRISM and PELOD scores were calculated using an online calculator. Outcome measures were PICU length of stay, inotrope usage, multiorgan dysfunction, and survival. ACR was compared with mortality scores for predicting survival.

Results: Microalbuminuria was present in $79.8 \%$ with a median value of $85 \mathrm{mg} / \mathrm{g}$ (interquartile range, $41.5-254 \mathrm{mg} / \mathrm{g}$ ). A positive correlation was found between albumin-creatinine ratio and PICU length of stay $(P=0.013, r=0.271)$. Albumin-creatinine ratio was significantly associated with organ dysfunction $(P=$ $0.004)$ and need for inotropes $(P=0.006)$. Eight deaths were observed in the PICU. The area under the curve for mortality for albumin-creatinine ratio (0.822) was comparable to that for PRISM (0.928) and PELOD (0.877). Albumin-creatinine ratio $>109 \mathrm{mg} / \mathrm{g}$ predicted mortality with a sensitivity of $87.5 \%$ and specificity of $63.2 \%$.

Conclusion: Microalbuminuria is a good predictor of PICU outcomes comparable with mortality scores.

Key words: Albumins, Creatinine, Mortality, Pediatric intensive care units

\section{Key message}

Question: Does microalbuminuria predict mortality in pediatric intensive care unit?

Finding: Positive correlation was found between albumincreatinine ratio and pediatric intensive care unit stay, organ dysfunction and need of inotropes. Area under the receiver operating characteristic curve for albumin-creatinine ratio was comparable to mortality scores.

Meaning: Microalbuminuria is a good predictor of outcome in pediatric intensive care unit and is comparable with mortality scores.

\section{Introduction}

The prevalence of systemic inflammatory response syndrome (SIRS) is high among hospitalized children. The risk of SIRS developing into multiorgan dysfunction (MODS) is also high, which is a major cause of morbidity and mortality in children. ${ }^{1)}$ Hence early detection of SIRS, prognostication, and initiation of appropriate treatment is of utmost importance for ensuring better outcomes in children. ${ }^{2)}$

The scoring systems for mortality prediction are developed after sophisticated statistical modelling and require computer support. The scores require many variables. The calculation of these scores is very tedious and expensive. It also has the disadvantage of interobserver variation. ${ }^{3)}$ Hence a reliable, fast, inexpensive and a bedside prognostic marker is required.

In patients with SIRS, there is an associated alteration in endothelial cell membrane structures due to the cascade of cytokines released. ${ }^{4)}$ It leads to albuminuria of varying degrees which is not detected by dipstick method. The early presence of microalbuminuria (MA) reflects the degree of insult and organ dysfunction. ${ }^{5,6}$ MA is estimated by spot urine albumincreatinine ratio (ACR). ${ }^{7}$

Data on the role of MA as a prognostic marker in children is

Corresponding author: Suchetha S. Rao, MD. Department of Paediatrics, Kasturba Medical College, Mangalore, Manipal Academy of Higher Education, Manipal Karnataka, India 
limited.

In this study, an attempt was made to understand the role of MA at admission in predicting the outcome of the pediatric patients admitted to pediatric intensive care unit (PICU) in comparison with mortality scores - PRISM and PELOD.

\section{Methods}

A cross-sectional study was conducted at teaching hospitals between January 2015 to October 2016. Pediatric patients were enrolled by convenient sampling after obtaining Kasturba Medical college, Mangalore Institutional Ethics Committee approval (approval number: IEC KMC MLR 11-14/230) and informed consent.

The inclusion criteria were all children admitted to the PICU and need for PICU stay for more than 24 hours. Patients with acute kidney injury, anuria, urologic trauma resulting in frank haematuria, failure to collect urine samples, existing chronic renal disease, urinary infection or receiving antibiotic or nephrotoxic drugs were excluded. Demographical, clinical, and laboratory details were entered in a structured proforma. Blood investigations and spot urine sample were collected at admission. MA was estimated by spot urine ACR. Urinary albumin was quantified by immunoturbidimetric test using a kit from certified reference material diagnostics system using semi autoanalyzer. The Jaffe method was used to calculate the urinary creatinine. The clinical scores-PRISM and PELOD—were calculated using Société française d'anesthésie et de reanimation calculator using the equation described by Pollack et al. ${ }^{8)}$ and Leteurtre et al. ${ }^{9}$ respectively.

PRISM score was computed with: Glasgow Coma Scale, heart rate, respiratory rate, blood pressure, pupillary reflexes, partial pressure of arterial oxygen $\left(\mathrm{PaO}_{2}\right)$ /fraction of inspiratory oxygen $\left(\mathrm{FiO}_{2}\right)$, partial pressure of arterial carbon dioxide $\left(\mathrm{PaCO}_{2}\right)$, bicarbonate, calcium, potassium, total bilirubin, glucose and prothrombin or partial thromboplastin time. ${ }^{7)}$

PELOD score was determined using heart rate, $\mathrm{PaO}_{2}, \mathrm{PaCO}_{2}$, Glasgow Coma Scale, pupillary reaction, mechanical ventilation status, total white cell count, platelet, creatinine, prothrombin time/international normalized ratio and serum glutamic oxaloacetic transaminase. ${ }^{7}$

Sample size of 82 was calculated with $z=1.96$ at $95 \%$ confidence level; expected sensitivity, $P=0.63 ; q=0.37$ and margin of error, $d=20 \%$ (POWER OF STUDY). Relevant details were entered in excel spread sheet and transferred to SPSS ver. 17 (SPSS Inc., Chicago, IL, USA). Descriptive data were expressed as mean, median, percentage and standard deviation. MannWhitney $U$ test was applied to compare variables between survivors and nonsurvivors. Spearman rank correlation was used to find the correlation between ACR and length of PICU stay. A $P<0.05$ was considered statistically significant. A receiver operating characteristic (ROC) curve was used to establish the validity of ACR compared to mortality scores for predicting the mortality. The outcome was assessed based on the length of PICU stay, inotrope requirement, the presence of MODS and survival of the patient.

\section{Results}

The study included 84 cases. The median age of the patients was 1.75 years (interquartile range [IQR], 0-5.75 years). Baseline characteristics of the study population are depicted in Table 1. Majority cases were respiratory which included bronchopneumonia and bronchiolitis (48.8\%), others were infectious diseases malaria, dengue and typhoid (25\%), cardiovascular (5.9\%), burns (5.9\%), and malignancies (4.8\%). Fever was present in majority of the patients (95\%), tachypnea and

Table 1. Baseline characteristics of the study population $(n=84)$

\begin{tabular}{lc}
\hline Characteristic & Value \\
\hline Age group & \\
1 Month-1 yr & $41(48.8)$ \\
$2-5$ Years & $21(25)$ \\
$6-12$ Years & $15(17.9)$ \\
$13-18$ Years & $7(8.3)$ \\
Sex & \\
Male & $51(60.7)$ \\
Female & $33(39.3)$ \\
Type of disease & \\
Respiratory & $41(48.8)$ \\
Infectious disease & $21(25.0)$ \\
Neurological & $8(9.5)$ \\
Cardiovascular & $5(5.9)$ \\
Burns & $5(5.9)$ \\
Malignancy & $4(4.8)$ \\
Pediatric intensive care unit stay (day) & $4.27 \pm 5.56$ \\
\hline
\end{tabular}

Values are presented as number (\%) or mean \pm standard deviation.

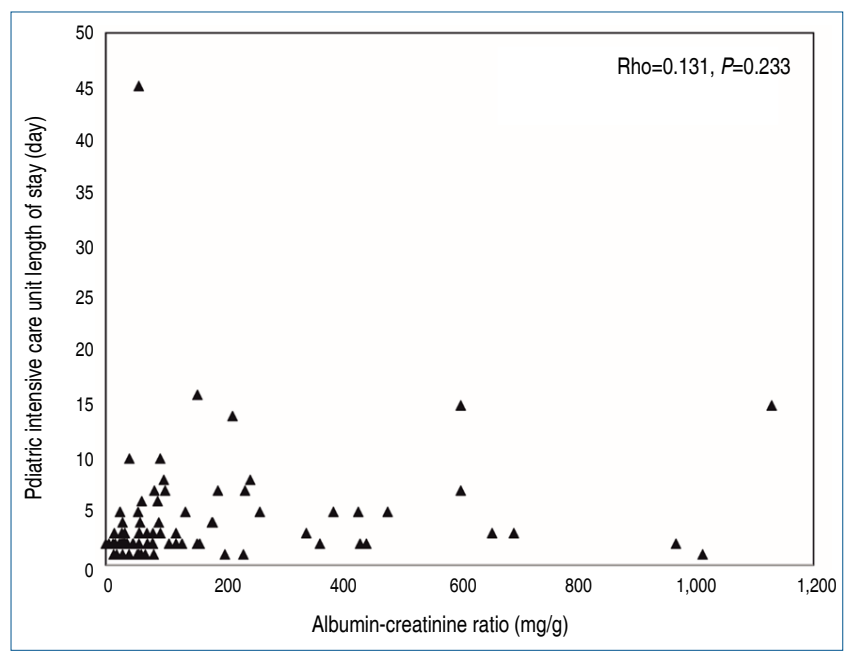

Fig. 1. Scatter plot of correlation between albumin-creatinine ratio $(\mathrm{mg} / \mathrm{g})$ on day 1 represented on the $x$-axis and pediatric intensive care unit length of stay (days) represented on $y$-axis. Spearman rank correlation was used to test correlation (Rho $=0.131, P=0.233$ ) 


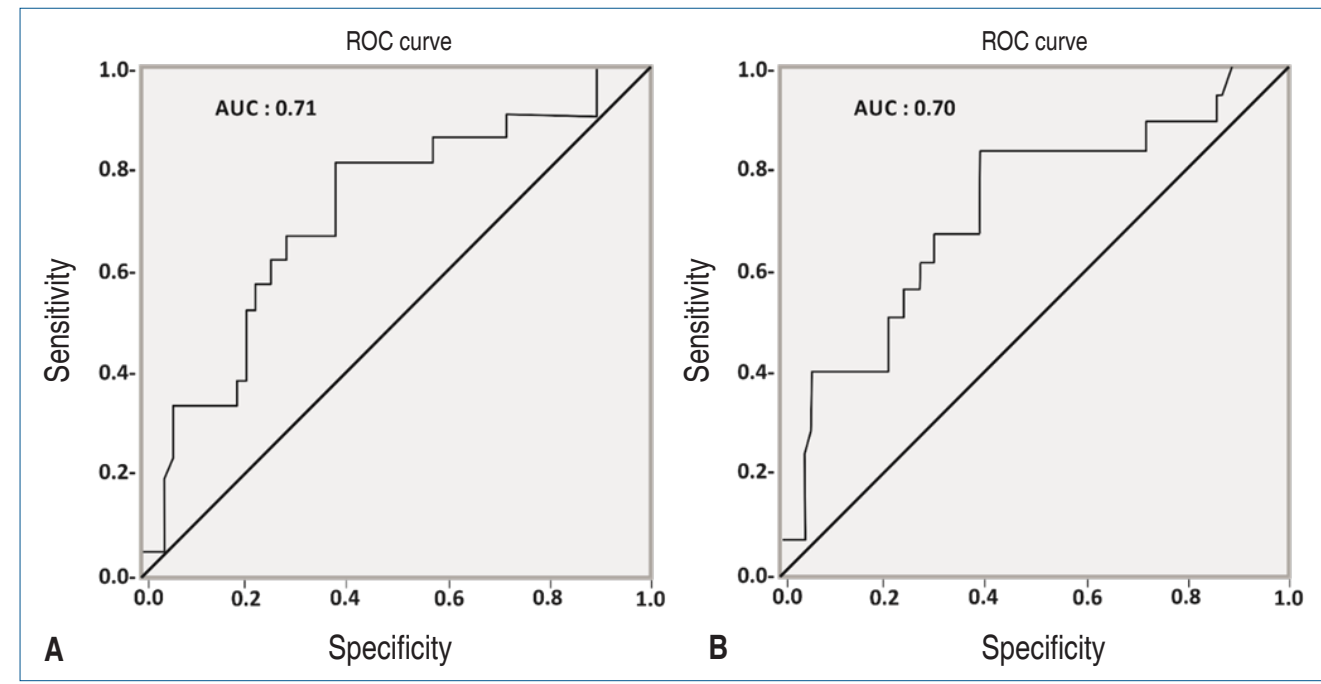

Fig. 2. Receiving-operating characteristic (ROC) curve of albumin-creatinine ratio $(\mathrm{mg} / \mathrm{g})$ on day 1 to predict multiorgan dysfunction (A) and inotrope use (B). AUC, area under the curve.

Table 2. Comparison of Pediatric Risk of Mortality scores and baseline variables used for calculating the score at admission to pediatric intensive care unit between survivors and nonsurvivors

\begin{tabular}{lccc}
\hline Variable & Survivor $(\mathrm{n}=76)$ & Nonsurvivor $(\mathrm{n}=8)$ & $P$ value \\
\hline Pediatric Risk of Mortality score & $3(1-5)$ & $14.5(8.25-20)$ & $<0.001$ \\
Systolic blood pressure $(\mathrm{mmHg})$ & $85(65-104)$ & $64(57-72)$ & \\
Diastolic blood pressure $(\mathrm{mmHg})$ & $55(46-68)$ & $137(40-54)$ & \\
Heart rate & $120(100-136)$ & $67(46-72)$ & \\
Respiratory rate & $41(30-56)$ & $267(170-365)$ & \\
$\mathrm{PaO} /$ FiO2 & $326(311.8-331.5)$ & $30(18.7-43.3)$ & \\
PaCO2 & $31.9(25-34.2)$ & $1.75(1.37-1.98)$ & \\
Prothrombin time (INR) & $1.25(1.1-1.4)$ & $0.4(0.3-0.9)$ & \\
Total bilirubin (mg/dL) & $0.67(0.5-0.8)$ & $7.85(7.3-9)$ & \\
Calcium (mg/dL) & $9(8.8-9.2)$ & $90(48-122)$ & \\
Potassium (meq/L) & $4.6(4.2-5)$ & $18(12.6-20)$ \\
Glucose (mg/dL) & $76(56-100)$ & $8(100)$ \\
Bicarbonate (meq/L) & $18(17-19)$ & $5(3-10)$ \\
Pupillary reaction & $76(100)$ & \\
$\quad$ Bilaterally equal and reacting & $12(11-13)$ & \\
Glasgow Coma Scale & &
\end{tabular}

Values are represented as median (interquartile range) or number (\%)

$\mathrm{PaO}_{2}$, partial pressure of oxygen; $\mathrm{FiO}_{2}$, fraction of inspired oxygen; $\mathrm{PaCO}_{2}$, partial pressure of carbon dioxide; INR, international normalized ratio.

The Mann-Whitney $U$ test was used to compare variables between survivors and nonsurvivors.

Boldface indicates a statistically significant difference with $P<0.05$.

tachycardia were seen in $36 \%$ and $31 \%$ of the subjects respectively. Prevalence of MA was in $79.8 \%$. The median value of MA was $85 \mathrm{mg} / \mathrm{g}$ (IQR, $41.5-254 \mathrm{mg} / \mathrm{g})$. A positive correlation was found between ACR and PICU stay $(P=0.233$, Rho=0.131) (Fig. 1).

In the study, 19 children (22\%) had the need for inotropes and $20(23 \%)$ had MODS. ACR showed highly significant association with the need for inotropes $(P=0.006)$ and Multiorgan dysfunction $(P=0.004)$. As per the ROC curves, area under the curve (AUC) derived. for multiorgan dysfunction was 0.71 (Fig. 2A) and the need for inotrope was 0.70 (Fig. 2B). The cutoff value hence generated was $89 \mathrm{mg} / \mathrm{g}$ which had a sensitivity of $83.3 \%$ for Inotrope use and $81 \%$ for multiorgan dysfunction. The specificity for this value for inotrope use and multiorgan dysfunction was $60.6 \%$ and $61.9 \%$, respectively.

Eight deaths were observed in PICU. The median length of stay for nonsurvivors in PICU was 5 days (IQR, 3-45 days). A significantly higher PRISM (Table 2), PELOD and ACR scores were found in the patients who died $(P<0.05)$ (Table 3$)$.

As per ROC curves, AUC derived for mortality for ACR was $0.822(0.711-0.934)$ which had sensitivity of $87.5 \%$ and specificity 63.2\%, AUC for PRISM was 0.928 (0.833-1.000) which had sensitivity $87.5 \%$ and specificity $92.1 \%$ and AUC for PELOD was 0.877 (0.726-1.000) which had sensitivity of 
Table 3. Comparison of Pediatric Logistic Organ Dysfunction scores, baseline variables used for calculating the score, and albumincreatinine ratio at admission to pediatric intensive care unit between survivors and nonsurvivors

\begin{tabular}{|c|c|c|c|}
\hline Variable & Survivor $(n=76)$ & Nonsurvivor $(n=8)$ & $P$ value \\
\hline Pediatric Logistic Organ Dysfunction score & $0(0)$ & $22(10.25-31.75)$ & $<0.001$ \\
\hline Heart rate & $120(100-136)$ & $137(106-169)$ & \\
\hline Systolic blood pressure (mmHg) & $85(65-104)$ & $64(57-72)$ & \\
\hline Glasgow Coma Scale & $12(11-13)$ & $5(3-10)$ & \\
\hline \multicolumn{4}{|l|}{ Pupillary reaction } \\
\hline Bilaterally equal and reacting & $76(100)$ & $8(100)$ & \\
\hline SGOT (UI/L) & $33(21-46)$ & $94(43.3-136.5)$ & \\
\hline Prothrombin time (INR) & $1.25(1.1-1.4)$ & $1.75(1.37-1.98)$ & \\
\hline $\mathrm{PaO}_{2}$ & $98(90-110.2)$ & $112.4(68-179.5)$ & \\
\hline $\mathrm{PaCO}_{2}$ & $31.9(25-34.25)$ & $30(18.7-43.3)$ & \\
\hline \multicolumn{4}{|l|}{ Mechanical ventilation } \\
\hline Yes & $0(0)$ & $4(50)$ & \\
\hline No & $76(100)$ & $4(50)$ & \\
\hline White blood cells (cells $/ \mathrm{mm}^{3}$ ) & $12,400(6,625-16,700)$ & $23,100(14,000-24,385)$ & \\
\hline Platelets (cells $/ \mathrm{mm}^{3}$ ) & $332,500(180,000-485,000)$ & $163,500(116,250-225,500)$ & \\
\hline Creatinine $(\mathrm{mg} / \mathrm{dL})$ & $0.3(0.3-0.5)$ & $0.5(0.3-0.7)$ & \\
\hline Albumin-creatinine ratio $(\mathrm{mg} / \mathrm{g})$ & $76.5(33.75-196.75)$ & $359(159.5-567.5)$ & 0.003 \\
\hline
\end{tabular}

Values are represented as median (interquartile range) or number (\%)

SGOT, serum glutamic oxaloacetic transaminase; INR, international normalized ratio; $\mathrm{PaO}_{2}$, partial pressure of oxygen; PaCO2, partial pressure of carbon dioxide.

The Mann-Whitney $\mathrm{U}$ test was used to compare variables between survivors and nonsurvivors.

Boldface indicates a statistically significant difference with $P<0.05$.

Table 4. Receiver-operating characteristic curve analysis of mortality scores and albumin-creatinine ratio at admission for mortality in the pediatric intensive care unit

\begin{tabular}{lcccc}
\hline Variable & Cutoff point & The area under the curve & Sensitivity (\%) & Specificity (\%) \\
\hline Pediatric Risk of Mortality score & 3.8 & $0.928(0.833-1)$ & 87.5 & 92.1 \\
Pediatric Logistic Organ Dysfunction score & 1.15 & $0.877(0.726-1)$ & 75 & 88.2 \\
Albumin-creatinine ratio (mg/g) & 109 & $0.822(0.711-0.934)$ & 87.5 & 63.2 \\
\hline
\end{tabular}

Values represent the area under curve of receiver-operating characteristic curve of the Pediatric Risk of Mortality score, Pediatric Logistic Organ Dysfunction score, and albumin-creatinine ratio (mg/g) for mortality. At the defined cutoff point, sensitivity, and specificity are described.

$75 \%$ and specificity of $88.2 \%$ (Table 4). The AUC for ACR was comparable to the AUC for standard mortality scores PRISM) and PELOD (Fig. 3). The cutoff point for ACR to predict mortality was derived-109 $\mathrm{mg} / \mathrm{g}$ with sensitivity and specificity of $87.5 \%$ and $63.2 \%$, respectively.

\section{Discussion}

Diffuse endothelial dysfunction in critically ill patients leads to increased systemic capillary permeability, renal component being manifested as MA. Levels of MA increase rapidly following inflammatory insult. ${ }^{10)}$ Studies have established MA as prognostic marker of morbidity and mortality in intensive care units. ${ }^{11)}$

In the present study, MA was present in $79.8 \%$ of cases and was significantly higher in nonsurvivors $(P=0.003)$. ACR had a highly significant association with the need of inotropic and presence of MODS with cutoff values $89 \mathrm{mg} / \mathrm{g}$ derived from the ROC curve. The ACR value of $109 \mathrm{mg} / \mathrm{g}$ was found to be comparable with the standard scores in predicting mortality with

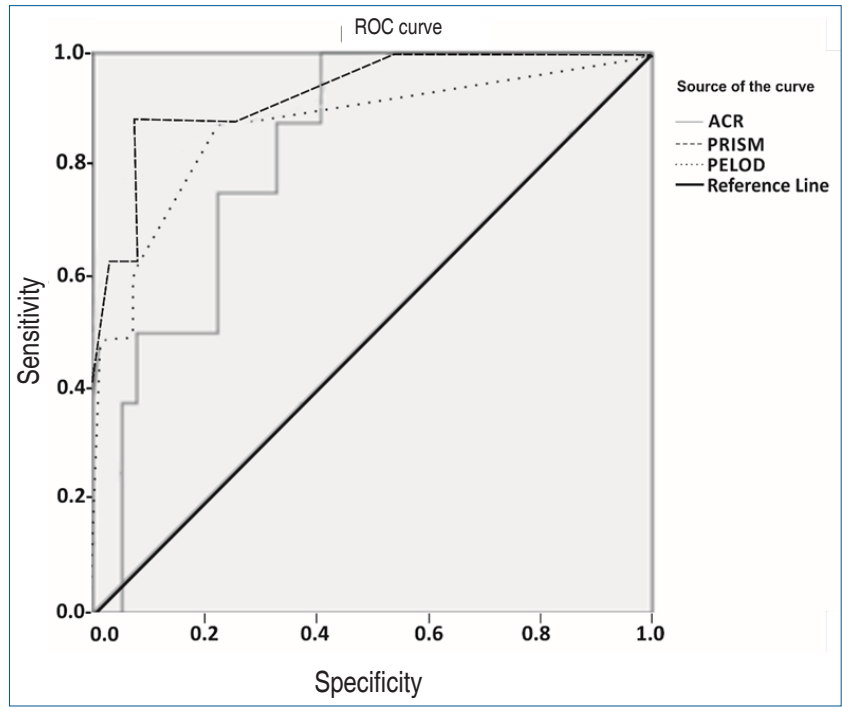

Fig. 3. Comparison of receiving-operating characteristic (ROC) curves of Pediatric Risk of Mortality (PRISM) score, Pediatric Logistic Organ Dysfunction (PELOD) score, and the albumin-creatinine ratio (ACR) $\mathrm{mg} / \mathrm{g}$ at admission to predict mortality. 
good sensitivity and specificity.

As per the study of Anil et al..$^{7)}$ done in pediatric age group, at admission ACR (ACR1) and at 24 hours (ACR2) with values of $34.2 \mathrm{mg} / \mathrm{g}$ and $31.6 \mathrm{mg} / \mathrm{g}$ generated from ROC had sensitivities of $63.3 \%$ and $80 \%$ and specificities of $93.3 \%$ and $66.7 \%$ for predicting mortality, respectively. They also found that ACR1 and ACR 2 correlated with use of inotropes, duration of mechanical ventilation and mortality. Studies involving pediatric patients are limited. A study by Saeed et al., ${ }^{12}$ in critically ill adult patients showed that increased ACR after 24 hours of admission correlated with the need of mechanical ventilation, vasoactive support and increased mortality. Serial ACR value and its prog. nostic significance were not estimated in the present study, hence effect of treatment was not determined.

Basu et al. ${ }^{10)}$ found that in adults admitted to intensive care unit (ICU). An ACR 2 value of $101 \mathrm{mg} / \mathrm{g}$ could predict ICU mortality with a sensitivity of $69 \%$ and specificity of $67 \%$. ACR 2 was concluded to be a good predictor of mortality as the APACHE II score. It was associated with length of ICU stay and duration of mechanical ventilation as well.

A study done by Enadle ${ }^{13)}$ in critically ill adult patients, concluded that ACR at 24 hours of admission was better associated with length of ICU stay and glomerular filtration rate and outcome than ACR at admission. He also concluded that ACR could also comment on the patient's response toward the goaldirected therapy.

In a study of critically ill septic adults done by Tayeh et al., ${ }^{14)}$ it was found that ACR2 was significantly elevated in those who required mechanical ventilation and inotropic support $(P=$ 0.01 and $P=0.009$ ). it was found that ACR2 had AUC of 0.88 for mortality with a cut off value of $110.5 \mathrm{mg} / \mathrm{g}$ with $100 \%$ sensitivity and $86 \%$ specificity.

Studies have shown that the absence of MA at 24 hours was suggestive of improved ICU survival and comparable to APACHE II scores. ${ }^{15,16)}$

To conclude, ACR was found to have a significant role in predicting inotrope use, organ dysfunction and had a good correlation with PICU stay. It was also found that ACR in nonsurvivors at admission was significantly higher and is comparable in predicting the mortality in SIRS to the clinical mortality scores. Thus, ACR is a good one-time and reliable tool to predict the need for inotropes, the presence of MODS and mortality. Future studies are required to evaluate the correlation of serial measurement of ACR with treatment and outcome in patients with SIRS in PICU. An intervention study could also be done based on ACR levels to reduce morbidity and mortality. Early ionotropic therapy would possibly prevent MODS and thereby reduce morbidity and enhance early recovery.

\section{Conflicts of interest}

No potential conflict of interest relevant to this article was reported.

\section{Acknowledgments}

Dr. Kamalakshi Bhat, Professor, MD Paediatrics, Department of Paediatrics, Kasturba Medical College Mangalore, Manipal Academy of Higher Education for her constant encouragement and guidance throughout this study.

\section{References}

1. Pavare J, Grope I, Gardovska D. Prevalence of systemic inflammatory response syndrome (SIRS) in hospitalized children: a point prevalence study. BMC Pediatr 2009;9:25.

2. Galley HF. Bench-to-bedside review: targeting antioxidants to mito chondria in sepsis. Crit Care 2010;14:230.

3. Gulla KM, Sachdev A. Illness severity and organ dysfunction scoring in pediatric intensive care unit. Indian J Crit Care Med 2016;20:27-35.

4. Singh A, Satchell SC. Microalbuminuria: causes and implications. Pediatr Nephrol 2011;26:1957-65.

5. Thorevska N, Sabahi R, Upadya A, Manthous C, Amoateng-Adjepong Y. Microalbuminuria in critically ill medical patients: prevalence, predictors, and prognostic significance. Crit Care Med 2003;31:1075-81.

6. Gosling P, Czyz J, Nightingale P, Manji M. Microalbuminuria in the intensive care unit: Clinical correlates and association with outcomes in 431 patients. Crit Care Med 2006;34:2158-66.

7. Anil AB, Anil M, Yildiz M, Kamit Can F, Bal A, Gokalp G, et al. The importance of microalbuminuria in predicting patient outcome in a PICU. Pediatr Crit Care Med 2014;15:e220-5.

8. Pollack MM, Ruttimann UE, Getson PR. Pediatric risk of mortality (PRISM) score. Crit Care Med 1988;16:1110-6.

9. Leteurtre S, Martinot A, Duhamel A, Gauvin F, Grandbastien B, Nam TV, et al. Development of a pediatric multiple organ dysfunction score: use of two strategies. Med Decis Making 1999;19:399-410.

10. Basu S, Chaudhuri S, Bhattacharyya M, Chatterjee TK, Todi S, Majumdar A. Microalbuminuria: an inexpensive, non invasive bedside tool to predict outcome in critically ill patients. Indian J Clin Biochem 2010; 25:146-52.

11. Gopal S, Carr B, Nelson P. Does microalbuminuria predict illness severity in critically ill patients on the intensive care unit? A systematic review. Crit Care Med 2006;34:1805-10.

12. Saeed MA, Mahdy RE, Mohammed SA. Urine albumin/creatinine ratio as an early predictor of outcome in critically ill patients with sepsis. Res Opin Anesthesia Intensive Care 2018:5:267-78.

13. Enadle R. A study of micro albuminuria in predicting the outcome of critically ill patients. MedPulse - Int Med J 2015;2:525-8.

14. Tayeh O, Taema KM, Eldesouky MI, Omara AA. Urinary albumin/ creatinine ratio as an early predictor of outcome in critically-ill septic patients. Egypt J Crit Care Med 2016;4:47-55.

15. Basu S, Bhattacharya M, Chatterjee TK, Chaudhuri S, Todi SK, Majumdar A. Microalbuminuria: a novel biomarker of sepsis. Indian J Crit Care Med 2010;14:22-8.

16. Vohra I, Singh A, Ali S, Yadav M, Mahdi AA. Analysis of microalbuminuria to predict sensitivity and outcome of traumatic intensive care unit patients. Int J Recent Sci Res 2016;7:12310-4. 